\title{
High heterogeneity of plasma membrane microfluidity in multidrug-resistant cancer cells
}

\author{
Céline Boutin* \\ Université de Technologie de Troyes \\ Laboratoire de Nanotechnologie et \\ d'Instrumentation Optique \\ LRC CEA, Institut Charles Delaunay \\ FRE CNRS 2848 \\ 12 rue Marie Curie, BP 2060 \\ Troyes Cedex 10010, France
}

\author{
Yann Roche ${ }^{*,+}$ \\ Université de Reims Champagne-Ardennes \\ Matrice Extracellulaire Dynamique Cellulaire \\ UMR CNRS 6237 \\ Faculté de Pharmacie \\ 51 rue Cognacq-Jay \\ Reims Cedex 51096, France
}

\section{Christine Millot}

Université de Reims Champagne-Ardennes Matrice Extracellulaire Dynamique Cellulaire UMR CNRS 6237

Faculté de Pharmacie

51 rue Cognacq-Jay

Reims Cedex 51096, France

\section{Régis Deturche}

\section{Pascal Royer}

Université de Technologie de Troyes

Laboratoire de Nanotechnologie et d'Instrumentation Optique

LRC CEA, Institut Charles Delaunay

FRE CNRS 2848

12 rue Marie Curie, BP 2060

Troyes Cedex 10010, France

\section{Michel Manfait}

Université de Reims Champagne-Ardennes

Matrice Extracellulaire Dynamique Cellulaire

UMR CNRS 6237

Faculté de Pharmacie

51 rue Cognacq-Jay

Reims Cedex 51096, France

\section{Jérôme Plain}

Université de Technologie de Troyes

Laboratoire de Nanotechnologie et

d'Instrumentation Optique

LRC CEA, Institut Charles Delaunay

FRE CNRS 2848

12 rue Marie Curie, BP 2060

Troyes Cedex 10010, France

\author{
Pierre Jeannesson \\ Jean-Marc Millot \\ Université de Reims Champagne-Ardennes \\ Matrice Extracellulaire Dynamique Cellulaire \\ UMR CNRS 6237 \\ Faculté de Pharmacie \\ 51 rue Cognacq-Jay \\ Reims Cedex 51096, France
}

\section{Rodolphe Jaffiol}

Université de Technologie de Troyes

Laboratoire de Nanotechnologie et

d'Instrumentation Optique

LRC CEA, Institut Charles Delaunay

FRE CNRS 2848

12 rue Marie Curie, BP 2060

Troyes Cedex 10010, France

\begin{abstract}
Diffusion-time distribution analysis (DDA) has been used to explore the plasma membrane fluidity of multidrug-resistant cancer cells (LR73 carcinoma cells) and also to characterize the influence of various membrane agents present in the extracellular medium. DDA is a recent single-molecule technique, based on fluorescence correlation spectroscopy (FCS), well suited to retrieve local organization of cell membrane. The method was conducted on a large number of living cells, which enabled us to get a detailed overview of plasma membrane microviscosity, and plasma membrane microorganization, between the cells of the same line. Thus, we clearly reveal the higher heterogeneity of plasma membrane in multidrug-resistant cancer cells in comparison with the nonresistant ones (denoted sensitive cells). We also display distinct modifications related to a membrane fluidity modulator, benzyl alcohol, and two revertants of multidrug resistance, verapamil and cyclosporin-A. A relation between the distribution of the diffusion-time values and the modification of membrane lateral heterogeneities is proposed. (0) 2009 Society of Photo-Optical Instrumentation Engineers. [DOI: $10.1117 / 1.3155518$ ]
\end{abstract}

Keywords: fluorescence correlation spectroscopy (FCS); membrane diffusion; multidrug-resistant cancer cells.

Paper 08370R received Oct. 14, 2008; revised manuscript received Feb. 17, 2009; accepted for publication Apr. 15, 2009; published online Jun. 23, 2009.

\section{Introduction}

The two-dimensional (2-D) fluid organization of cell membranes is a common crucial structure for every form of life. Plasma membrane fluidity is also strongly involved in numerous physiological processes of cells. Thus, the plasma membrane fluidity appears as a relevant biophysical parameter in the understanding of membrane-related biological mechanisms. Fluidity, and more particularly viscosity at the microscale of cell membrane, depends on the molecular dynamics

1083-3668/2009/14(3)/034030/8/\$25.00 (C) 2009 SPIE
${ }^{+}$Current address: Laboratoire Plantes-Microbe-Environnement, UMR INRA 1088/CNRS 5184, Université de Bourgogne, 17 rue Sully, 21065 Dijon, France. Address all correspondence to: Dr. Rodolphe Jaffiol. LNIO (Laboratoire de Nanotechnologie et d'Instrumentation Optique), LRC CEA, Institut Charles Delaunay FRE CNRS 2848, Université de Technologie de Troyes, 12 rue Marie Curie, BP 2060, 10010 Troyes cedex, France. Tel: +33 3 25718527; Fax: +33 25718456; E-mail: rodolphe.jaffiol@utt.fr. 
of its components (lipids, proteins) within the supramolecular 2-D assembly. These dynamic processes are rotational and transbilayer movements, flip-flop, and lateral motion. ${ }^{1}$ The plasma membrane dynamic is also strongly altered in the presence of lateral heterogeneities constraining the molecular dynamics. It is now assumed that these heterogeneities are related to a local organization of the plasma membrane, implying various domains characterized by differences in composition and packing, the so-called lipid rafts, corrals, and caveolaes, for examples. ${ }^{2,3}$ Lateral membrane heterogeneities are involved in various physiological processes, such as signaling, protein and lipid trafficking, cell growth, and cell adhesion. $^{4,5}$

Currently, fluorescence spectroscopy and imaging allow us to reveal the plasma membrane organization and fluidity through the motion of its constituents. ${ }^{6}$ Fluorescence anisotropy is widely used to reveal the fluidity through the rotational and translational movement of lipids, for example. Nevertheless, anisotropy experiments are often performed on a concentrated bulk sample, i.e., many cells are simultaneously studied, and such experiments provide only an ensembleaveraged measure of the membrane fluidity. On the contrary, fluorescence recovery after photobleaching (FRAP) is the usual way to study the lateral diffusion at the single-cell level. However, FRAP needs a high relative concentration of dye molecules, and unfortunately, these required high concentration conditions could strongly perturb the membrane dynamic. Therefore, single-molecule fluorescence spectroscopy techniques constitute a well-suited alternative to overcome this invasive aspect and averaging ensemble effects. Among the single molecule techniques, fluorescence correlation spectroscopy (FCS) is the relevant method to investigate the fluidity of biological membranes. ${ }^{7,8}$ The potential of FCS to relate heterogeneity in model and natural membranes was recently demonstrated. ${ }^{9,10}$ FCS measurements at different spatial scales were also done to probe membrane domains organization with so-called FCS diffusion laws. ${ }^{11-13}$ The latter should provide a suitable method to identify the diffusion behavior of proteins in the plasma membrane. Based on FCS, diffusion-time distribution analysis (DDA) is a recent technique proposed to analyze single-molecule diffusion and is well suited to study very locally heterogeneous samples, such as plasma membrane of living cells. ${ }^{14}$

Tumoral cells could present a multidrug resistance (MDR) to chemotherapeutic treatments. This drug resistance is mainly dependent on biomechanisms occurring at the plasma membrane level, and particularly on overexpression of ATPase-binding cassette (ABC) transporter, such as P-glycoprotein (Pgp). ${ }^{15,16}$ This resistance to drugs would be associated to membrane-mediated mechanisms involving modification of membrane fluidity, drug permeability, presence of microdomains (rafts, caveolae), and membrane proteins expression. All these membrane mechanisms can be monitored by FCS through the lateral diffusion of a fluorescent membrane probe. Some modulator agents, named revertants, have been developed in order to increase the intracellular concentration of cytotoxic drugs in MDR cells. Some of them are known to inhibit the Pgp activity. Other modulators as fluidizers seem to act by increasing the passive transmembrane motion (flip-flop) of drugs. ${ }^{17}$ Nevertheless, the interactions between the modulators and the plasma membrane and the exact mechanism of inhibition are still not clear.

In this paper, we propose to explore by DDA the plasma membrane fluidity of one multidrug-resistant cancer cell line (CHO-LR73 transfected with MDR1 cDNA) and the influence of three molecules added to the extracellular medium: two revertant agents (verapamil and cyclosporin-A) $)^{18,19}$ and a membrane fluidizer, benzyl alcohol. ${ }^{20,21}$

\section{Materials and Methods}

\subsection{Cell Culture and Sample Preparation}

Chinese hamster ovarian LR-73 drug-sensitive cells (LR73-S) have been transfected with the mammalian expression plasmid pDREX4 containing the biologically active mouse MDR1 cDNA insert of phage $\lambda$ DR11 (the drug-resistant cells, i.e., the transfected ones, are denoted LR73-R). The LR73-S and LR73-R cell lines were grown in RPMI-1640 medium (Gibco) supplemented with 10\% fetal calf serum (Gibco). Doxorubicin at a concentration of $10^{-7} \mathrm{M}$ was added to LR73-R culture medium to ensure against revertants. All experiments with resistant cell line were performed on growing cells that have been in drug-free medium for more than a week. Resistance index (IC50 of resistant cells/IC50 of control cells) was 45 (Ref. 22).

For FCS analysis, LR73 carcinoma cells were grown in sterile glass-based Petri dishes (Iwaki Glass) in RPMI 1640 medium with Glutamax (Gibco) enriched with $10 \%$ fetal calf serum (Gibco) at $37^{\circ} \mathrm{C}$ in a humidified atmosphere of $5 \%$ $\mathrm{CO}_{2}$. Last, cells were labeled with a $250-\mathrm{nM}$ solution of DiA [4-(4-(didecylamino) styryl)-N-methylpyridinum iodide, Molecular Probes] during $5 \mathrm{~min}$ in growth medium. FCS measurements were performed at room temperature in D-PBS (Gibco) in order to avoid the background signal related to the growth medium. When necessary, incubations in D-PBS (Gibco) supplemented with a pharmacological concentration of benzyl alcohol $(10 \mathrm{mM}),{ }^{17}$ verapamil $(5 \mu \mathrm{M}),{ }^{23}$ or cyclosporin-A $(5 \mu \mathrm{M})^{18}$ were done during $5 \mathrm{~min}$ at $37^{\circ} \mathrm{C}$.

\subsection{Preparation of Unilamellar Vesicles}

Giant unilamellar vesicles (GUVs) were prepared by using the rapid evaporation method. ${ }^{24}$ Briefly, $20 \mu \mathrm{L}$ of dioleoylphosphatidylcholine (DOPC) previously dissolved in chloroform $(0.1 \mathrm{M})$, were mixed in $980 \mu \mathrm{L}$ of chloroform, $150 \mu \mathrm{L}$ of methanol, and $7 \mathrm{~mL}$ of distilled water. The organic solvents have been removed in a rotary evaporator under reduced pressure. After evaporation, the resulting aqueous solution contained the GUVs. With such a method of synthesis, unilamellar vesicles are produced with typical diameter of some $10 \mu \mathrm{m}$. The GUVs were labeled with a $100-\mathrm{nM}$ solution of DiA during $45 \mathrm{~min}$. Glass substrates previously chemically functionalized using a silanization process with aminopropyldimethylethoxysilane (APDMS, 97\%), ${ }^{25}$ are used for confocal and FCS observations. After sedimentation, such kinds of substrate avoid GUV movement on the glass/water interface.

\subsection{FCS Optical Setup}

FCS was conducted with a home-made confocal optical device, which has been developed on an inverted Olympus microscope (IX70). This microscope is designed to probe very small volumes $\left(\approx 0.35 \mu \mathrm{m}^{3}\right)$ by controlling the spatial extent 


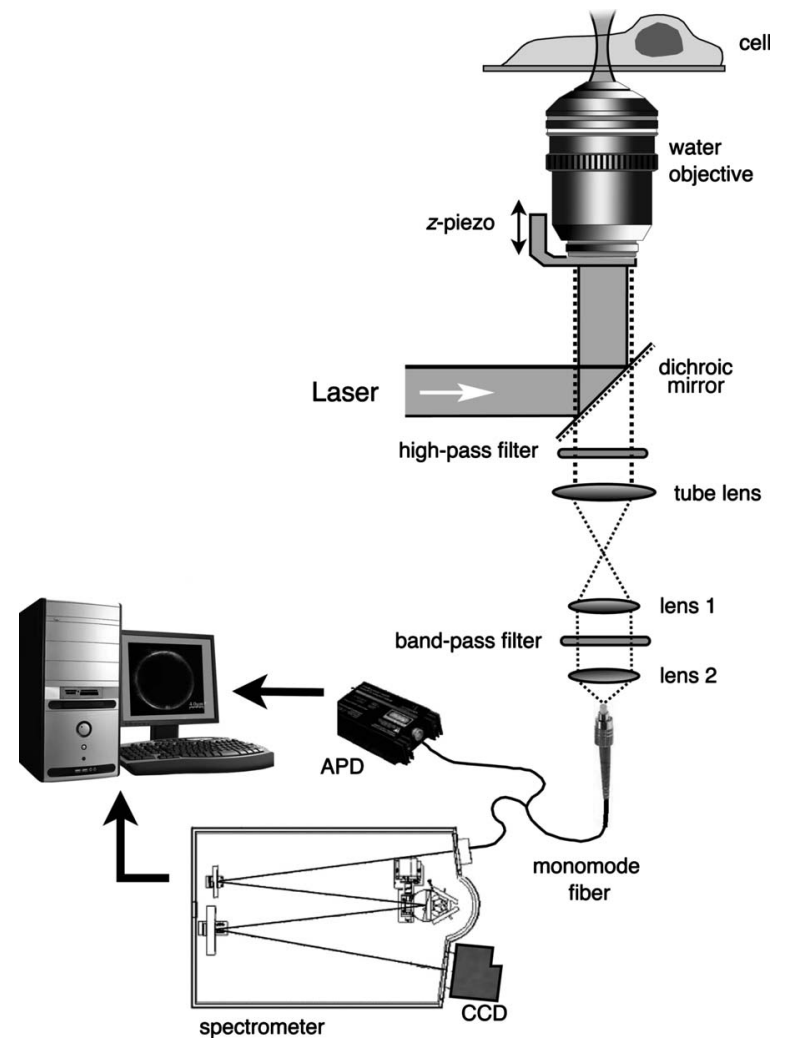

Fig. 1 Experimental setup (see text for details).

of the laser beam at the entrance pupil of the objective and the size of the pinhole detector. A schematic representation of the setup is presented in Fig. 1.

The excitation light is provided by the 488-nm argon laser line. After passing through a beam expander to sufficiently enlarge the laser beam at the entrance pupil of the objective, the laser beam is strongly focused through a high numerical aperture water immersion objective (Olympus UPlanSApo $60 \times, \mathrm{NA}=1.2$ ). In all experiments, the laser power, measured at the back pupil aperture of the objective, is kept constant to $100 \mu \mathrm{W}$. The fluorescence emission is collected through the same objective and passes through a sharp high-pass filter over $500 \mathrm{~nm}$ (Semrock LP02-488RU) and a highly selective bandpass filter centered at $560 \mathrm{~nm}$, with a bandwidth of $25 \mathrm{~nm}$ (Semrock FF01-560/25). According to the total magnification of our microscope, $M=15(60 \times$ for the objective and $0.25 \times$ for the additional optical imaging part comprise lenses 1 and 2: see Fig. 1), a monomode optical fiber with a core diameter of $9 \mu \mathrm{m}$ is used as point detector for the confocal detection. ${ }^{26}$ Last, the fluorescence signal $F(t)$ is recorded with an avalanche photodiode (PerkinElmer APD, SPCM-AQR-16: see Fig. 1), and the fluorescence fluctuations are analyzed through the temporal autocorrelation function (ACF) of $F(t)$-namely, the ACF, denoted $G(\tau)$. The APD is connected to a homemade single-photon counting module, ${ }^{27}$ and a computational multi- $\tau$ procedure calculates the autocorrelation functions $G(\tau)$ with a quasi-geometrical progression of the lag time from $0.2 \mu$ s to $\sim 3$ s (Ref. 28).

FCS experiments have been performed at the single-cell level using the so-called Z-scan method previously proposed

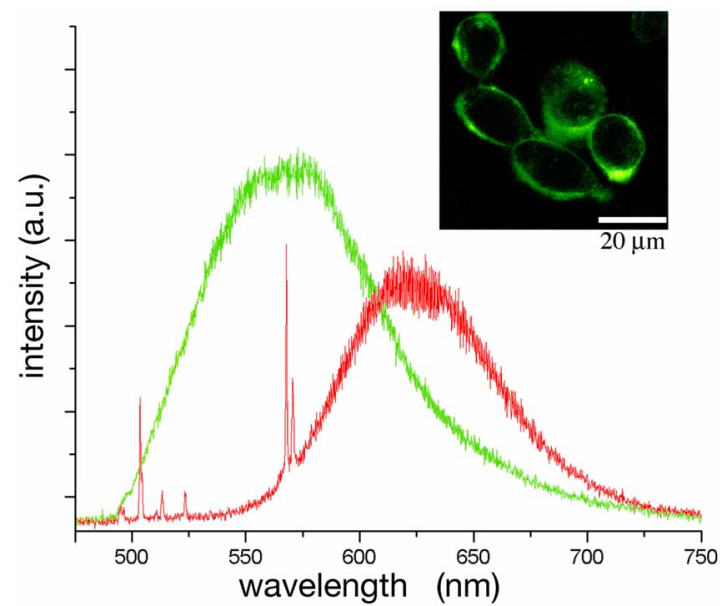

Fig. 2 Fluorescence spectra emission: Red curve for a nanomolar concentration of DiA in DMSO (the narrow lines correspond to the Raman scattering of DMSO); green curve for DiA into the plasma membrane of LR73 cells (see text for labeling conditions). The fluorescence spectrum of DiA in DMSO is similar to the one in water, with an emission maximum $\approx 620 \mathrm{~nm}$ in DMSO and $\approx 610 \mathrm{~nm}$ in water. Inset: Typical confocal fluorescence picture of LR73-S labeled with DiA. (Color online only.)

by A. Benda et al. ${ }^{29,30}$ The Gaussian-like profile of the focusing laser beam gives rise to an axial dependence (zdependence) of the diffusion time of DiA in the membrane, denoted $\tau_{d}^{2 D}$. Consequently, a minimum value of $\tau_{d}^{2 D}$ appears when the smallest size of the beam radius - namely, the beam waist - is exactly located on the plasma membrane. Hence, the accurate values of the membrane diffusion time $\tau_{d}^{2 D}$ (i.e., the slowest ones) are obtained according to a fine achievement of the axial positioning of the observation volume with a z-piezo system (PIFOC device from PI, Fig. 1). ${ }^{25}$

The fluorescence signal, $F(t)$, can also be sent to a Jobin Yvon spectrometer (TRIAX 550, Fig. 1), and confocal fluorescence observations were performed with a Bio-Rad MRC1024 system.

\section{Results}

\subsection{Analysis of Membrane Autocorrelation Functions}

To ensure that the fluorescent probe location and behavior are appropriate for FCS experiments, confocal observations and spectral analysis were made on DiA-labeled LR73 cells. The inset in Fig. 2 clearly shows that fluorescence from DiA is mostly located at the plasma membrane level. The distribution of fluorescence along the membrane is quite homogenous and does not vary throughout observation; in particular, no dye internalization is noted (data not shown). Moreover, a $\approx 50$-nm blue shift of the DiA emission is observed between a DiA solution and the DiA in the cell membrane, as shown in Fig. 2. This blue shift is the spectral fingerprint of the well fluorophore insertion in the plasma membrane. ${ }^{31}$ Thus, these two combined observations ensure that DiA labeling of LR73 cells strictly stains the plasma membrane.

Membrane autocorrelation functions $G(\tau)$ are fitted with the following model describing the simultaneous translational diffusions of both a slow (2-D) and a fast (3-D) component, 
according to the molecules diffusing within the plasma membrane and residual contribution of DiA molecules freely diffusing in the vicinity of the membrane, respectively: $:^{32,33}$

$$
\begin{aligned}
G(\tau)= & 1+\left[1+\frac{F \exp \left(\frac{-\tau}{\tau_{F}}\right)}{1-F}\right] \times\left[\frac{\left\langle c r^{2 D}\right\rangle^{2}}{\left\langle c r^{\text {total }}\right\rangle^{2}} \frac{1}{\left\langle N^{2 D}\right\rangle}\left(1+\frac{\tau}{\tau_{d}^{2 D}}\right)^{-1}\right. \\
& +\frac{\left(\left\langle c r^{\text {total }}\right\rangle-\left\langle c r^{2 D}\right\rangle\right)^{2}}{\left\langle c r^{\text {total }}\right\rangle^{2}} \frac{1}{\left\langle N^{3 D}\right\rangle}\left(1+\frac{\tau}{\tau_{d}^{3 D}}\right)^{-1} \\
& \left.\times\left(1+\frac{\tau}{S^{2} \tau_{d}^{3 D}}\right)^{-1 / 2}\right] .
\end{aligned}
$$

The first term of Eq. (1) depicts, for a fraction $F$, the DiA molecules in a nonfluorescent state, which appears for singlettriplet dynamics, ${ }^{34}$ and also for membrane association/ dissociation kinetics. ${ }^{35}$ The characteristic time related to such nonfluorescent processes is named $\tau_{F}$. The second term describes the 2-D diffusion of the DiA in the membrane. The last term represents the residual contribution of fluorescent molecules freely diffusing (3-D process) near the plasma membrane. $\left\langle N^{2 D}\right\rangle$ and $\left\langle N^{3 D}\right\rangle$ are the average numbers of diffusing molecules for the 2-D diffusion and the 3-D diffusion, respectively. $c r^{\text {total }}$ and $c r^{2 D}$ are the respective fluorescence signals (denoted counting rate, $c r$ ) of the total amount of DiA and only for molecules diffusing within the plasma membrane. The respective diffusion times for each class are $\tau_{d}^{2 D}$ and $\tau_{d}^{3 D} . S$ is the axial elongation of the observation volume $(S$ is typically $\approx 5)$. In our cell samples, the DiA is largely present in the plasma membrane (Fig. 2), and the minimum of $\tau_{d}^{2 D}$ (assessed by the Z-scan method) always occurs together with a maximum of the total fluorescence signal, $c r^{\text {total }}$. In this way, we can evaluate very precisely the diffusion time of DiA molecules within the plasma membrane.

To reduce the noise on $G(\tau)$ and thus increase the accuracy of data evaluation, each FCS measurement results from the average of three autocorrelation curves, recorded consecutively at the same position on the cell membrane, each during $20 \mathrm{~s}$ (Ref. 28). Moreover, in all experiments, the laser power, measured at the back pupil aperture of the objective, is kept constant to $100 \mu \mathrm{W}$. Two typical autocorrelation curves (ACFs) fitting on living LR73 sensitive and resistant cells are presented in Fig. 3(a) and 3(b). (All these ACFs were evaluated by a standard least squares procedure.) On living cells, we show that in all cases, a two-component fit, according to Eq. (1), is necessary. On the contrary, a pure 2-D diffusion of DiA is recorded in GUV [Fig. 3(c)]. In the case of two diffusing species, the fast diffusive component is compatible with DiA freely diffusing in D-PBS medium. Thus, this fast diffusion time is kept fixed in the analysis to the value obtained from preliminary study in D-PBS, $\tau_{d}^{3 D} \approx 70 \mu \mathrm{s}$. The 2-D diffusion time $\tau_{d}^{2 D}$ and $\left\langle c r^{2 D}\right\rangle$ thus appear as the only free-fit parameters to be precisely determined by Eq. (1).

\subsection{Membrane Diffusion-Time Distribution Analysis from Sensitive and Resistant Cells}

We first aim to compare the lateral diffusion of the amphiphile membrane probe DiA in drug-resistant $(\mathrm{R})$ and drug-sensitive (S) LR73 ovarian carcinoma cells. FCS measurements were

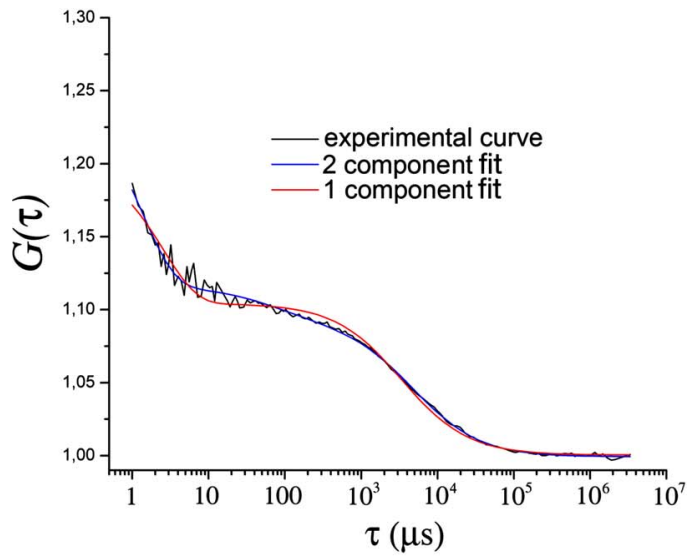

(a)

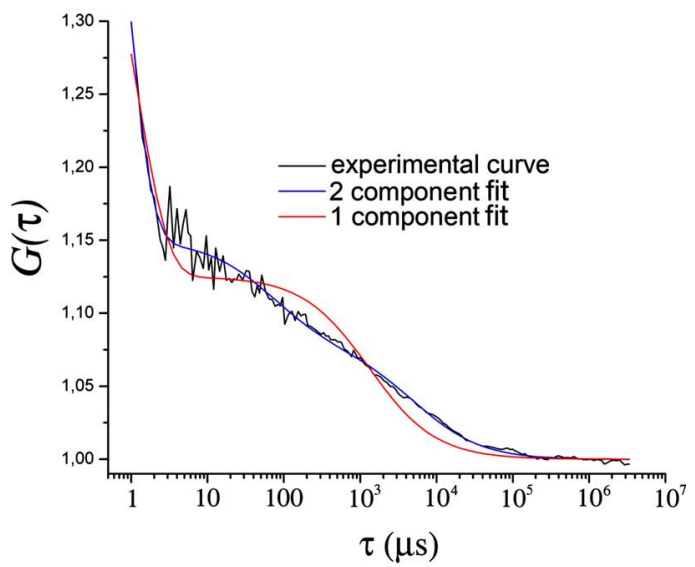

(b)

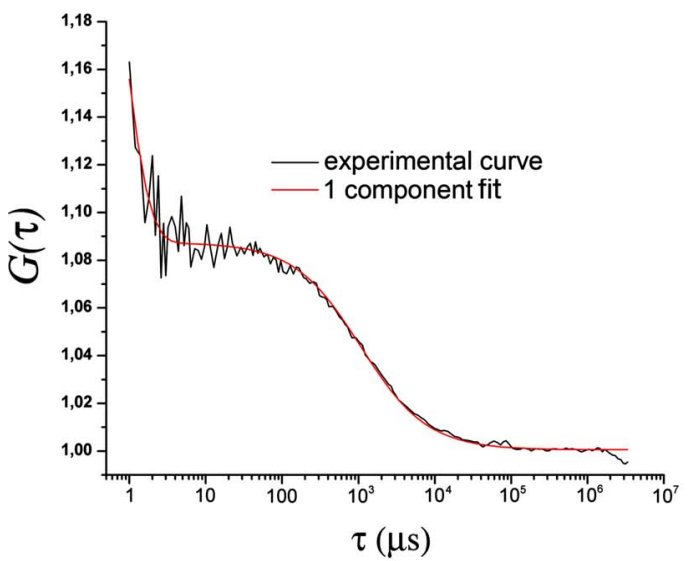

(c)

Fig. 3 (a) and (b): Two typical experimental autocorrelation functions (ACFs) obtained for DiA-labeled LR73 cells (see text for labeling conditions). (c): Typical ACF recorded for a giant unilamellar vesicle (GUV) labeled with DiA (see text for labeling conditions). Black curves: Experimental autocorrelation curves. Blue curves: Total fit according to Eq. (1). Red curves: Fit according to a single class of molecules diffusing in two dimensions. (Color online only.)

carried out for LR73-S and LR73-R populations of, respectively, 110 cells. The distribution plots of the diffusion times, $\tau_{d}^{2 \mathrm{D}}$, i.e., the diffusion-time distribution, are represented as counting histograms (Fig. 4). 


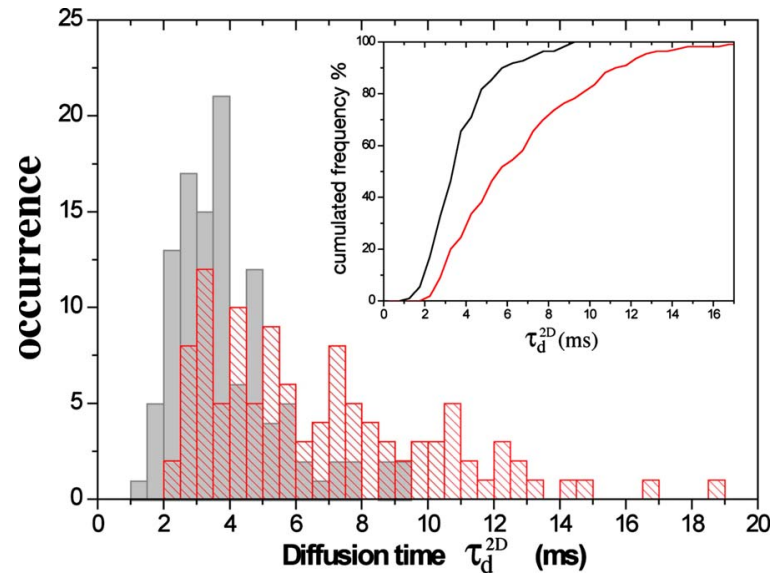

Fig. 4 Diffusion-time distributions obtained through a cell-by-cell measurement, from two populations of 110 LR73-cells each, sensitive line (gray columns) and resistant line (red-striped columns) (see text for labeling conditions). These histograms have been built with a column bin size equal to the highest error (i.e., highest uncertainty) assessed through the autocorrelation curve fitting, here $0.5 \mathrm{~ms}$. Inset: The corresponding cumulative frequency profiles for LR73-S (black line) and LR73-R (red line). (Color online only.)

This representation highlights the extensive dispersion of the two studied $\tau_{d}^{2 \mathrm{D}}$ datasets and clearly exhibits a broad distribution for the LR73-R resistant cell line. Both distributions are asymmetric and not mean-centered, showing a significant skewness toward longer times (this effect being more marked for LR73-R cells). The inset in Fig. 4 compares the cumulated frequency profiles for the LR73-S and LR73-R diffusion-time distributions. ${ }^{36}$ In addition, these profiles display some distinct noteworthy features: a sharp progression reaching 100\% at about $10 \mathrm{~ms}$ for the LR73-S dataset and a flat profile, ending around $18 \mathrm{~ms}$, in the case of LR73-R cells. Interestingly, the comparison of cumulated frequency profiles reveals than for LR73-R, $\approx 20 \%$ of the measured $\tau_{d}^{2 \mathrm{D}}$ values are higher than $10 \mathrm{~ms}$, whereas LR73-S cells present diffusion-time values below this threshold.

Mean values of the diffusion-time distribution, denoted $\left\langle\tau_{d}^{2 \mathrm{D}}\right\rangle$, are $3.9 \mathrm{~ms}$ versus $6.7 \mathrm{~ms}$ for LR73-S and LR73-R cells, respectively (Table 1). Results show a marked difference, as confirmed by the nonparametric Wilcoxon test ( $p$ $<0.001)$. The dispersion of the $\tau_{d}^{2 \mathrm{D}}$ measurements is assessed through the standard deviations of diffusion-time distribution, noted $\sigma_{\tau_{d}^{2 \mathrm{D}}}$, which are $1.6 \mathrm{~ms}$ and $3.4 \mathrm{~ms}$ for LR73-S and LR73-R cells, respectively, as shown in Table 1. The strong difference of $\sigma_{\tau_{d}^{2}}$ between both cell lines is validated through the F-test comparison for equal variance $(p<0.001)$.

In summary, the lateral membrane diffusion behavior of DiA is clearly distinct between sensitive LR73 cell lines and resistant ones. The latter exhibit a broad diffusion-time distribution, an increased mean value, and a significant fraction of slow diffusion events $\left(\tau_{d}^{2 \mathrm{D}}>10 \mathrm{~ms}\right)$.

\subsection{Fluidity Modulation Assessed with DDA}

The lateral diffusion of DiA in the plasma membrane of LR73-S and LR73-R cells was also investigated by FCS in the presence of two kinds of compounds present in the extracellular medium: membrane-perturbing agent benzyl alcohol (BA), and two MDR reverting drugs (verapamil and cyclosporin-A). Cumulated frequency profiles are deduced from FCS measurements done in each condition (benzyl alcohol, verapamil, and cyclosporin-A). Figure 5(a) compares the profiles obtained for LR73-S cells untreated (red curve) and treated with BA (black curve). The presence of BA leads to a narrower and short-time-shifted distribution of the diffusion times, as shown in Fig. 5(a). Influence of benzyl alcohol is characterized by important modifications of the mean values

Table 1 Statistical parameters estimated from the distribution of diffusion times $\left(\tau_{d}^{2 D}\right)$ and the distribution of diffusion coefficients $\left(D^{2 D}\right)$ for GUVs, LR73-S, and LR73-R cell lines, with and without treatment. The diffusion coefficients $\left(D^{2 D}\right)$ were evaluated according to the relation $\tau_{d}^{2 D}=\omega_{o}^{2} / 4 D^{2 D}$, where $\omega_{o}$ is the $1 / \mathrm{e}^{2}$ radial width of the observation volume. It was determined to be $\omega_{0}=275 \pm 10 \mathrm{~nm}$ by using the experimental method described in Ref. 37.

\begin{tabular}{|c|c|c|c|c|c|}
\hline & & $\tau_{d}^{2 D}(\mathrm{~ms}$ & $D^{2 D}(\mu$ & & \\
\hline & $\mathrm{Me}$ & alue & Stanc & iation & \\
\hline & $\left\langle\tau_{d}^{2 D}\right\rangle$ & $\left\langle D^{2 D}\right\rangle$ & $\sigma_{\tau_{d}^{2 D}}^{2 D}$ & $\sigma_{D^{2 D}}$ & $\begin{array}{c}\text { Percentage beyond } \\
\qquad 10 \mathrm{~ms}\end{array}$ \\
\hline LR73-S untreated & 3.9 & 5.6 & 1.6 & 2.2 & 0 \\
\hline LR73-S + benzyl alcohol & 2.0 & 10.5 & 0.6 & 3.7 & 0 \\
\hline LR73-R untreated & 6.7 & 3.6 & 3.4 & 1.8 & 18 \\
\hline LR73-R + benzyl alcohol & 2.9 & 7.0 & 0.7 & 2.4 & 0 \\
\hline LR73-R + verapamil & 5.5 & 3.6 & 1.0 & 0.8 & 0 \\
\hline LR73-R + cyclosporin-A & 5.8 & 3.7 & 2.1 & 1.4 & 3 \\
\hline GUV & 1.2 & 16.4 & 0.2 & 2.3 & 0 \\
\hline
\end{tabular}




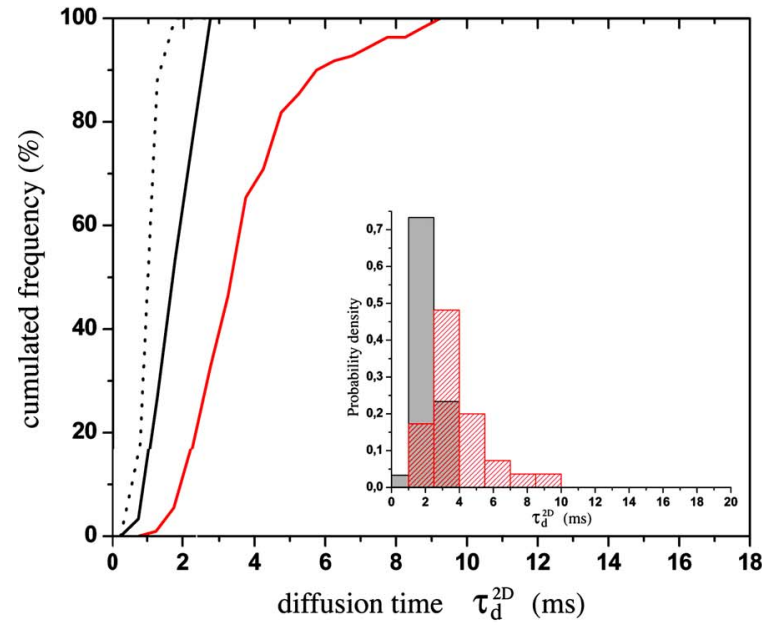

(a)

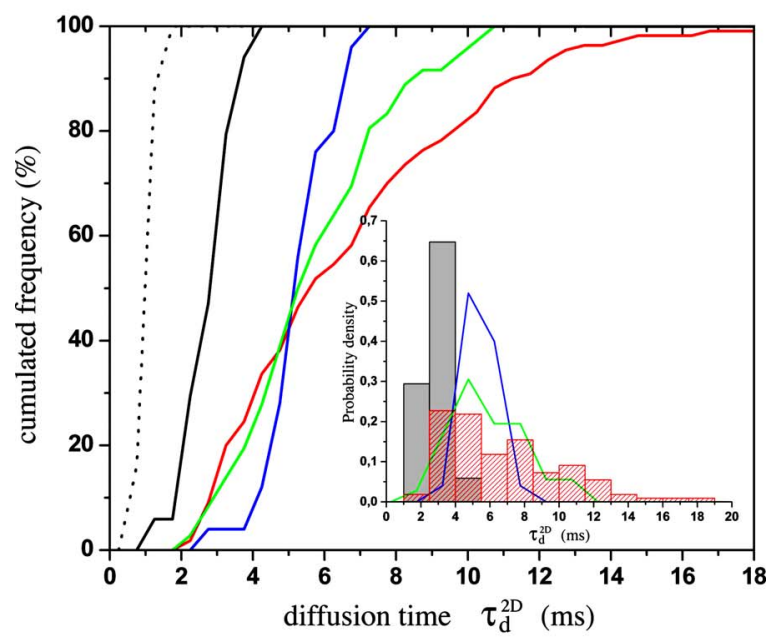

(b)

Fig. 5 Diffusion-time distributions and cumulative frequency profiles of LR73 cells and effect of membrane agents. (a): Diffusion-time cumulative-frequency profiles for LR73-S untreated (red line) and treated with benzyl alcohol (10 nM); (black line). Inset: The corresponding normalized diffusion-time distributions with the same color code (column bin size is $1.5 \mathrm{~ms}$ ). (b): Cumulative frequency profiles for LR73-R untreated (red line), treated with BA (10 mM, 5 min; black line), verapamil (5 $\mu \mathrm{M}, 5 \mathrm{~min}$; blue line), and cyclosporin-A (5 $\mu \mathrm{M}$, 5 min green line). Inset: The corresponding normalized diffusion-time distributions with the same color code. Diffusion-time cumulative frequency profiles for GUVs are also plotted in each graphic (black dottes curves). (Color online only.)

from $3.9 \mathrm{~ms}$ to $2.0 \mathrm{~ms}(p<0.001$; nonparametric Wilcoxon test) and by the standard deviation decrease from 1.6 to $0.6 \mathrm{~ms}(p<0.001$; F-test $)$, as shown in Table 1 . The cumulative frequency profiles obtained for treated or untreated LR73-R cells are presented in Fig. 5(b). Benzyl alcohol still exerts a marked effect on the diffusion-time distribution, simultaneously correlated to a strong decrease of mean and standard deviation, as shown in Table 1. These differences are confirmed by nonparametric Wilcoxon test and F-test for equal variance ( $p<0.001$ for both tests).

The influence of the verapamil drug is less marked but leads to a sharper profile and abolishes the diffusing events higher than $10 \mathrm{~ms}$, which are typical of untreated LR73-R cells [Fig. 5(b)]. In the presence of cyclosporin, the cumulative profile is slightly affected, with a partial conservation of diffusion events around $10 \mathrm{~ms}$ [Fig. 5(b)]. Concurrently, verapamil gives a stronger diminution of $\sigma_{\tau_{d}^{2 D}}$ than cyclosporin, as shown in the inset in Fig. 5(b) and in Table 1. F-testing indicates that these variances are significantly different $(p<0.01)$. Interestingly, both reverting drugs exert weak effects on the mean value, as can be seen in Table 1, and nonparametric Wilcoxon tests attest that $\tau_{d}^{2 \mathrm{D}}$ distributions for treated (verapamil or cyclosporin) LR73-cells are not significantly shifted (Table 1).

In summary, verapamil, cyclosporin-A, and benzyl alcohol exert concrete modulations on the diffusion behavior of $\mathrm{DiA}$ in LR73 cells. The addition of benzyl alcohol in extracellular medium induces a significant increase of the plasma membrane fluidity and reduces considerably its heterogeneity, tending; toward phospholipids vesicle behavior (i.e., behavior of a pure lipid bilayer membrane) see Fig. 5 and Table 1. MDR reverting drugs keep the mean value, $\left\langle\tau_{d}^{2 \mathrm{D}}\right\rangle$, quite constant, and only verapamil totally abolishes the slow-diffusion events $\left(\tau_{d}^{2 \mathrm{D}}>10 \mathrm{~ms}\right)$.

\section{Discussion}

The degree of motion of hydrocarbon chains within the lipid bilayer is known as membrane fluidity. Previous observations have shown differences in structural order of the lipid phase of the plasma membrane between sensitive and resistant cells ${ }^{38}$ and that a decrease of membrane fluidity was associated with the degree of resistance to drugs. ${ }^{39}$ More recently, fluorescence anisotropy measurements with DPH dyes established an inhibition of Pgp transport activity by increasing the plasma membrane fluidity. ${ }^{40}$ Therefore, our results can be discussed regarding the membrane composition of sensitive and resistant cells, and also with respect to the role of the lipid organization on P-glycoprotein function.

\subsection{Membrane Lipid Composition in Sensitive and Resistant Cells}

Membrane fatty acid composition showed that resistant cells contain, in comparison to sensitive cells, a low amount of unsaturated fatty acid and on the contrary a high proportion of saturated fatty acids. ${ }^{41-43}$ Moreover, it was shown that unsaturated fatty acids addition increase the fluidity of the plasma membrane, which emphasizes the role of unsaturated fatty acids on the fluidity. Furthermore, in comparison with sensitive cells, esterified cholesterol and triglycerides are also present in a high proportion in two MDR cells lines derived from MCF-7 and LoVo. ${ }^{44}$ Recent FCS studies on model lipid bilayer have clearly pointed out slow diffusion times in the presence of saturated fatty acids, cholesterol, and triglycerides. $^{45,46}$ Therefore, the presence of differences in terms of fluidity between sensitive and resistant cells can be obviously related to the lipid composition of the plasma membrane (Fig. 4).

This high diversity of lipids in a cell membrane (in comparison to a model membrane) gives rise to the membrane heterogeneity, the so-called microdomains such as lipid rafts and caveolae. ${ }^{47}$ Membrance microdomains consist of a model of compact and ordered association of cholesterol and sphingolipids with long and saturated fatty acids, "floating" in a 
more fluid phase. ${ }^{4,5}$ Lipid microdomains are present in both sensitive and resistant cells, but some of them are specific to multidrug-resistant cells. These specific microdomains give rise to the visible differences between both sensitive and resistant diffusion-time distributions, as shown in Fig. 4.

P-glycoprotein exists in raft and nonraft membrane domains, in proportions depending on the cell types. A large amount of the total Pgp cellular content was analyzed in detergent-resistant membrane regions from MDR cell lines. ${ }^{48}$ A correlation between Pgp function and its association to lipid rafts components has been recently reported. ${ }^{49} \mathrm{P}$-glycoprotein also has enzymatic properties, which are associated with a less fluid and ordered membrane environment. ${ }^{50}$ To improve our understanding of the presence of Pgp in microdomains, we have begun FCS studies combined with their Z-scan method $^{30}$ to perform DDA at different spatial scale, as previously proposed by Lenne et al. ${ }^{12,13}$ By analysis of the FCS diffusion law of labeled Pgp, it should be possible to identify the repartition of Pgp in the plasma membrane. These complementary analyses are currently under way and are beyond the scope of this paper.

\subsection{Effects of P-Glycoprotein Modulators}

We show for both cell lines that the fluidizing effect of benzyl alcohol leads to an important increase of the plasma membrane fluidity (Fig. 5), with respect to a broad homogenization of the plasma membrane micro-organization, hence by the strong reduction of diffusion-time distribution width (assessed through the standard deviation; see Table 1). Like benzyl alcohol, many other molecules such as linoleic acid, chloroform, diethyl ether, detergents, and surfactants are known to fluidize the entire cell membrane. These molecules can also suppress the P-glycoprotein activity, as assayed either by cell efflux, by drug uptake in vesicles, or by ATPase activity. Thus, the increase of membrane fluidity may affect functions of membrane proteins, such as P-glycoprotein.

Changes in multidrug resistance by resistance modifiers such as verapamil would modify the fluidity of the cell membrane. A lower degree of structural order in cell membrane and an increased membrane fluidity was described in the verapamil-treated resistant $\mathrm{CH}(\mathrm{R}) \mathrm{C} 5$ subline. ${ }^{39}$ In contrast, verapamil induces no effect on the cell membrane fluidity of sensitive cells.

At the opposite of this nonspecific action of benzyl alcohol, revertant agents such as verapamil and cyclosporin-A, do not change entirely the fluidity of multidrug-resistant cells, as shown in Fig. 5(b). Just a few parts of the diffusion-time distribution are clearly affected. Consequently, these revertant agents would seem to act only on some specific microdomains of MDR cells. However, both cyclosporin-A and verapamil are probably competitive inhibitors of P-glycoprotein, and their effects on membrane fluidity are a side effect, not directly relevant to the reversal of the P-glycoprotein activity. Nevertheless, a reasonable hypothesis is that these specific microdomains observed in resistant cells constitute the highly structured environment, which probably contains, among other species, the P-glycoprotein. Modulator agents of the Pgp (verapamil and cyclosporine-A) homogenize the diffusiontimes toward the sensitive cell values, as shown in Fig. 5. Indeed, the inhibition of P-glycoprotein, known as a lipid translocase, could increase the membrane fluidity by disturbing the direct environment of the protein.

\section{Conclusion}

We report in this paper, for the first time to our knowledge, changes of membrane fluidity in resistant cells by means of a single molecule technique based on fluorescence correlation spectroscopy: diffusion-time distribution analysis (DDA). DDA, conducted at the single-cell level and on a large number of cells, provides complementary information about the plasma membrane fluidity between cells of the same cell line. Indeed, two significant pieces of information have been extracted from diffusion-time distributions: the average value, which is directly related to fluidity degree of the cell line, and the standard deviation, which gives information about the plasma membrane heterogeneity (i.e., the degree of structural order of plasma membrane). Consequently, we report a lower degree of fluidity for resistant cells, and more interestingly, a larger range of lateral diffusion times, as compared with sensitive cells. Other studies reported in the literature, using FRAP, fluorescence anisotropy, or electron spin resonance spectroscopy, have also revealed a lower fluidity for resistant cells than sensitive ones. ${ }^{38,39,42,51}$ However, the broad distribution of diffusion times recorded on MDR cells that can be obviously directly related to the high heterogeneity of its membrane, in term of organization, would be a new property of resistant cells. Besides this global property of MDR cells, the presence of distinct slow-diffusion sites $\left(\tau_{d}^{2 \mathrm{D}}>10 \mathrm{~ms}\right)$ linked to the resistance has been revealed through the action of fluidizer and revertant agents. These slow-diffusion sites observed in MDR cells may be related to specific microdomains, which probably contains, among other species, the P-glycoprotein.

\section{Acknowledgments}

This work was supported by the French National Research Agency (Contract No. ANR-JC07_190015), the Région Champagne-Ardenne (under the contract Projet Émergence 2006-2007 and for postdoctoral fellowship), and the Ligue Contre le Cancer (Comité de l'Aube).

\section{References}

1. J. Slavik, Fluorescent Probes in Cellular and Molecular Biology, CRC Press, Boca Raton, FL (1994).

2. F. R. Maxfield, "Plasma membrane microdomains," Curr. Opin. Cell Biol. 14, 483-487 (2002).

3. D. Marguet, P.-F. Lenne, H. Rigneault, and H.-T. He, "Dynamics in plasma membrane: how to combine fluidity and order," $E M B O \mathrm{~J} . \mathbf{2 5}$, 3446-3457 (2006).

4. K. Simons and E. Ikonen, "Functional rafts in cell membranes," $\mathrm{Na}$ ture (London) 387(6633), 569-572 (1997).

5. L. Rajendran and K. Simons, "Lipid rafts and membrane dynamics," J. Cell. Sci. 118, 1099-1102 (2005).

6. M. Hof, R. Hutterer, and V. Fidler (Eds.), Fluorescence Spectroscopy in Biology (Advanced Methods and Their Applications to Membranes, Proteins, DNA, and Cells), Springer-Verlag, Berlin (2005).

7. P. Schwille, U. Haupts, S. Maiti, and W. W. Webb, "Molecular dynamics in living cells observed by fluorescence correlation spectroscopy with one- and two-photon excitation," Biophys. J. 77, 22512265 (1999).

8. S. A. Kim, K. G. Heinze, and P. Schwille, "Fluorescence correlation spectroscopy in living cells," Nat. Methods 4(11), 963-973 (2007)

9. N. Kahya, D. Scherfeld, K. Bacia, and P. Schwille, "Lipid domain formation and dynamics in giant unilamellar vesicles explored by fluorescence correlation spectroscopy," J. Struct. Biol. 147, 77-89 
(2004).

10. S. Chiantia, N. Kahya, and P. Schwille, "Raft domain reorganization driven by short- and long-chain ceramide: a combined AFM and FCS study," Langmuir 23, 7659-7665 (2007).

11. L. Wawerezinieck, H. Rigneault, D. Maguet, and P.-F. Lenne, "Fluorescence correlation spectroscopy diffusion laws to probe the submicron cell membrane organization," Biophys. J. 89, 4029-4042 (2005).

12. P.-F. Lenne, L. Wawrezinieck, F. Conchonand, O. Wurtz, A. Boned, X.-J. Guo, H. Rigneault, H.-T. He, and D. Marguet, "Dynamics molecular confinement in the plasma membrane by microdomains and cytoskeleton meshwork," EMBO J. 25, 3245-3256 (2006).

13. J. Wenger, F. Conchonaud, J. Dintinger, L. Wawrezinieck, T. W. Ebbesen, H. Rigneault, D. Marguet, and P.-F. Lenne, "Diffusion analysis within single nanometric apertures reveals the ultrafine cell membrane organization," Biophys. J. 92, 913-919 (2007).

14. H. Jankevics, M. Prummer, P. Izewska, H. Pick, K. Leufgen, and H. Vogel, "Diffusion-time distribution analysis reveals characteristic ligand-dependent interaction patterns of nuclear receptor in living cells," Biochemistry 44, 11676-11683 (2005).

15. C. F. Higgins, "ABC transporters: from microorganisms to man," Аnпu. Rev. Cell Biol. 8, 67-113 (1992).

16. M. M. Gottesman, "Mechanisms of cancer drug resistance," Аnnu. Rev. Med. 53, 615-627 (2002)

17. R. Regev, Y. G. Assaraf, and G. D. Eytan, "Membrane fluidization by ether, other anesthetics, and certain agents abolishes P-glycoprotein ATPase activity and modulates efflux from multidrug-resistant cells," Eur. J. Biochem. 259, 18-24 (1999).

18. T. Tsuruo, H. Lida, S. Tsukagoshi, and Y. Sakurai, "Increased accumulation of vincristine and adriamycin in drug-resistant P388 tumor cells following incubation with calcium antagonists and calmodulin inhibitors," Cancer Res. 42, 4730-4733 (1982).

19. L. M. Slater, P. Sweet, M. Stupecky, and S. Gupta, "Cyclosporin A reverses vincristine and daunorubucin resistance in acute lymphatic leukemia in vitro," J. Clin. Invest. 77, 1405-1408 (1986).

20. J. Reyes and R. Latorre, "Effect of the anesthetics benzyl alcohol and chloroform on bilayers made from monolayers," Biophys. J. 28, 256280 (1979)

21. N. Shigapova, Z. Torok, G. Balogh, P. Goloubinoff, L. Vigh, and I. Horvath, "Membrane fluidization triggers membrane remodeling which affects the thermotolerance in Escherichia coli," BBRC 328, 1216-1223 (2005).

22. J. Dufer, C. Millot-Broglio, Z. Oum'Hamed, F. Liautaud-Roger, P. Joly, A. Desplaces, and C. Jardillier, "Nuclear DNA content and chromatin texture in multidrug-resistant human leukemic cell lines," Int. J. Cancer 60(1), 108-114 (1995).

23. B. Larrivée and D. A. Averill, "Modulation of adriamycin cytotoxicity and transport in drug-sensitive and multidrug-resistant Chinese hamster ovary cells by hyperthermia and cyclosporin A," Cancer Chemother. Pharmacol. 45, 219-230 (2000).

24. A. Moscho, O. Orwar, D. T. Chiu, B. P. Modi, and R. N. Zare, "Rapid preparation of giant unilamellar vesicles," Proc. Natl. Acad. Sci. U.S.A. 93, 11443-11447 (1996).

25. C. Boutin, R. Jaffiol, J. Plain, and P. Royer, "Surface modified single molecules free-diffusion evidenced by fluorescence correlation spectroscopy," J. Fluoresc. (2008, in press).

26. T. Wilson, "Fluorescence imaging in fiber-optic based confocal scanning microscope," Opt. Commun. 96, 133-141 (1993).

27. R. Jaffiol, Y. Blancquaert, A. Delon, and J. Derouard, "Spatial fluorescence cross-correlation spectroscopy," Appl. Opt. 45, 1225-1235 (2006).

28. T. Wohland, R. Rigler, and H. Vogel, "The standard deviation in fluorescence correlation spectroscopy," Biophys. J. 80, 2987-2999 (2001).

29. A. Benda, M. Benes, V. Marecek, A. Lhotsky, W. Th. Hermens, and M. Hof, "How to determine diffusion coefficients in planar phospholipid systems by confocal fluorescence correlation spectroscopy," Langmuir 19, 4120-4126 (2003).

30. J. Humpolickova, E. Gielen, A. Benda, V. Fagulova, J. Vercammen, M. Vandeven, M. Hof, M. Ameloot, and Y. Engelborghs, "Probing diffusion laws within cellular membranes by Z-scan fluorescence correlation spectroscopy," Biophys. J. 91, L23-25 (2006).

31. L. M. Loew and L. L. Simpson, "Charge-shift probes of membrane potential: a probable electrochromic mechanism for p-aminostyrlpyridinium probes on a hemispherical lipid bilayer," Biophys. J. 34, 353-365 (1981).
32. Y. Ohsugi, K. Saito, M. Tamura, and M. Kinjo, "Lateral mobility of membrane-binding proteins in living cells measured by total internal reflection fluorescence correlation spectroscopy," Biophys. J. 91, 3456-3464 (2006).

33. O. Krichevsky and G. Bonnet, "Fluorescence correlation spectroscopy: the technique and its applications," Rep. Prog. Phys. 65, 251297 (2002).

34. J. Widengren, U. Mets, and R. Rigler, "Fluorescence correlation spectroscopy of triplet states in solution: a theoretical and experimental study," J. Phys. Chem. 99, 13368-13379 (1995).

35. A. M. Lieto, R. C. Cush, and N. L. Thompson, "Ligand-receptor kinetics measured by total internal reflection with fluorescence correlation spectroscopy," Biophys. J. 85, 3294-3302 (2003).

36. G. E. P. Box, W. G. Hunter, and J. S. Hunter, Statistics for Experiments: An Introduction to Design, Data Analysis, and Model Building, John Wiley \& Sons, New York (1978).

37. J. Plain, R. Jaffiol, G. Lerondel, and P. Royer, "Porous surface statistical characterization via fluorescence correlation spectroscopy," Phys. Status Solidi A 204, 1507-1511 (2007).

38. A. Ramu, D. Glaubiger, I. T. Magrath, and A. Joshi, "Plasma membrane lipid structural order in Doxorubicin-sensitive and -resistant P388 cells," Cancer Res. 43, 5533-5537 (1983).

39. J. A. Siegfried, K. A. Kennedy, A. C. Sartorelli, and T. R. Tritton, "The role of membranes in the mechanism of action of the antineoplastic agent adriamycin. Spin-labeling studies with chronically hypoxic and drug-resistant tumor cells," J. Biol. Chem. 258(1), 339-343 (1983).

40. C. Cai, H. Zhu, and J. Chen, "Overexpression of caveolin-1 increases plasma membrane fluidity and reduces P-glycoprotein function in Hs578T/Dox," Biochem. Biophys. Res. Commun. 30, 868-874 (2004).

41. U. N. Das, N. Madhavi, G. Sravan Kumar, M. Padma, and P. Sangeetha, "Can tumour cell drug resistance be reversed by essential fatty acids and their metabolites?," Prostaglandins, Leukotrienes Essent. Fatty Acids 58, 39-54 (1998).

42. J. H. Dolderer, G. Zimmer, B. G. Woodcock, H. Bockhorn, R. Bickeboller, and H. Schuldes, "Resistance modulation in $\mathrm{CHO}$ cells by R-verapamil and bile salts is associated with physical and chemical changes in the cell membrane," Int. J. Clin. Pharmacol. Ther. 4, 196-203 (2000).

43. L. Le Moyec, R. Tatoud, A. Degeorges, C. Calabresse, G. Bauza, M. Eugène, and F. Calvo, "Proton nuclear magnetic resonance spectroscopy reveals cellular lipids involved in resistance to adriamycin and taxol by the K562 leukemia cell line," Cancer Res. 56, 3461-3467 (1996).

44. M. T. Santini, R. Romano, G. Rainaldi, P. Filippini, E. Bravo, L. Porcu, A. Motta, A. Calcabrini, S. Meschini, P. L. Indovina, and G. Arancia, "The relationship between ${ }^{1} \mathrm{H}-\mathrm{NMR}$ mobile lipid intensity and cholesterol in two human tumor multidrug resistant cell lines (MCF-7 and LoVo)," Biochim. Biophys. Acta 1531, 111-131 (2001).

45. D. Scherfeld, N. Kahya, and P. Schwille, "Lipid dynamics and domain formation on model membranes composed of ternary mixtures of unsaturated and saturated phosphatidylcholines and cholesterol," Biophys. J. 85, 3758-3768 (2003).

46. N. Kahya and P. Schwille, "How phospholipid-cholesterol interactions modulate lipid lateral diffusion, as revealed by fluorescence correlation spectroscopy," J. Fluoresc. 16, 671-678 (2006).

47. Y. Lavie and M. Liscovitch, "Changes in lipid and protein constituents of rafts and caveolae in multidrug resistant cancer cells and their functional consequences," Glycoconjugate J. 17, 253-259 (2000).

48. S. Barakat, L. Gayet, G. Dayan, S. Labialle, A. Lazar, V. Oleinikov, A. W. Coleman, and L. G. Baggetto, "Multidrug-resistant cancer cells contain two populations of P-glycoprotein with differently stimulated P-gp ATPase activities: evidence from atomic force microscopy and biochemical analysis," Biophys. J. 388, 563-571 (2005).

49. Z. Basco, H. Nagy, K. Goda, L. Bene, F. Fenyvesi, J. Matko, and G. Szabo, "Raft and cytoskeleton associations of an $\mathrm{ABC}$ transporter P-glycoprotein," Cytometry, Part A 61A, 105-116 (2004).

50. S. Orlowski, S. Martin, and A. Escargueil, "P-glycoprotein and "lipid rafts:" some ambiguous mutual relationships (floating on them, building them or meeting them by chance?)," Cell. Mol. Life Sci. 63, 1038-1059 (2006).

51. X. Liang and Y. Huang, "Physical state changes of membrane lipids in human lung adenocarcinoma $\mathrm{A}_{549}$ cells and their resistance to cisplatin," Int. J. Biochem. Cell Biol. 34, 1248-1255 (2002). 